\title{
Editorial
}

\section{A.L. Copley Best Paper Prize 2016}

\author{
Friedrich Jung ${ }^{\mathrm{a}}$, Philippe Connes ${ }^{\mathrm{b}, \mathrm{c}, \mathrm{d}}$ and Christian Lehmann ${ }^{\mathrm{e}}$ \\ ${ }^{a}$ Institute of Biomaterial Science and Berlin-Brandenburg Center for Regenerative Therapies, \\ Helmholtz-Zentrum Geesthacht, Teltow, Germany \\ ${ }^{\mathrm{b}}$ Laboratoire Interuniversitaire de Biologie de la Motricité (LIBM) EA7424, Equipe \\ “Biologie vasculaire et du globule rouge”, Université Claude Bernard Lyon 1, COMUE, Lyon, France \\ ${ }^{\mathrm{c}}$ Laboratoire d'Excellence sur le globule rouge (Labex GR-Ex), Paris, France \\ ${ }^{\mathrm{d}}$ Institut Universitaire de France, Paris, France \\ ${ }^{\mathrm{e} D e p a r t m e n t ~ o f ~ A n e s t h e s i a, ~ P a i n ~ M a n a g e m e n t ~ a n d ~ P e r i o p e r a t i v e ~ M e d i c i n e, ~ D a l h o u s i e ~ U n i v e r s i t y, ~}$ \\ Halifax, NS, Canada
}

The Editor-in-Chief and Editorial Board of Clinical Hemorheology and Microcirculation (CHM), as well as the Publisher (IOS Press) have decided to set an annual prize, named the A.L. Copley Best Paper Prize, to recognize the best article published every year in CHM, starting in 2016.

This prize has been named in honor of the Journal's Founding Editor, Professor Alfred Lewin Copley. A.L. Copley was a German-American medical scientist and an artist at the New York School in the fifties. He introduced the word "hemorheology" in 1952 which he defined as follows: "Hemorheology is concerned with deformation and flow properties of cellular and plasmatic components of blood in macroscopic, microscopic and submicroscopic dimensions, and with the rheological properties of the vessel structure which directly comes in contact with blood" (Copley, 1952). With G. Seaman, A.L. Copley widened the definition of hemorheology such as: "Hemorheology is also the study of the interaction of blood or its components and the vascular system with added foreign materials, such as drugs, plasma expanders, or prosthetic devices. Thus, hemorheology is the study of how the blood and the blood vessels can function and interact as parts of the living organism" (Copley and Seaman, 1981).

First of all the editorial team carefully read and noted all original articles published in 2016 and we wish to thank all authors. The criteria for selection include: originality and innovation, theoretical contribution, clarity of writing and presentation, and expected impact. A group of three editors was elected by the editorial board to find the best paper in a multistage process. Each of the three editors listed the best 12 papers published 2016 of his choice. From these 36 papers the editors looked for manuscripts which have been nominated independently by more than one editor (first stage). This was the case for 10 out of the 36 papers. Out of these 10 papers each editor chose what he considered the best three papers and allocated 5 points to the best of the three, 3 points to the second best and 1 point to the third. The total points were added for each paper, thus allowing the papers to be ranked. The three highest-ranked papers were:

P. Napoleão, T. Freitas and C. Saldanha, Effect of oxidized LDL on erythrocyte nitric oxide metabolism. Clin Hemorheol Microcirc 64 (2016), 971-975. doi: 10.3233/CH-168025. 
H. Yagi, H. Sumino, T. Aoki, K. Tsunekawa, O. Araki, T. Kimura, M. Nara, T. Ogiwara and M. Murakami, Impaired blood rheology is associated with endothelial dysfunction in patients with coronary risk factors. Clin Hemorheol Microcirc 62 (2016), 139-150. doi: 10.3233/CH-151960.

T. Neumann, M. Baertschi, W. Vilser, S. Drinda, M. Franz, A. Bruckmann, G. Wolf and C. Jung, Retinal vessel regulation at high altitudes. Clin Hemorheol Microcirc 63 (2016), 281-292. doi: $10.3233 / \mathrm{CH}-162041$.

Finally, the article with the highest amount of points ( $n=11$ points) which received the prize was the manuscript from Thomas Neumann and colleagues (team of Prof. Dr. Christian Jung, University Düsseldorf) for their work on the regulation of retinal vessels under either normobaric or hypobaric hypoxia conditions. The authors set up a two parts study where 1) healthy individuals were exposed to a simulated altitude of 5500 meters and 2) a second group of healthy individuals were brought to a mountain station at an altitude of 3000 meters. In the second part of the study, individuals were also treated with a dual endothelin (ET) receptor antagonist that binds the two ET receptor subtypes, $\mathrm{ET}_{\mathrm{A}}$ and $\mathrm{ET}_{\mathrm{B}}$. The authors investigated the retinal vessel diameter, response to flicker light, retinal oxygen saturation and retinal venous pressure in the different experimental conditions. Both hypoxic exposures caused an increase of retinal arterial and venous diameters and a decrease of the arterial and venous response to flicker light. In the second part of the study, retinal venous pressure increased in 6 individuals after ascent to 3000 meters and normalized after dual ET receptor antagonist. This finding clearly shows that hypoxia may disturb retinal vascular reactivity in apparently healthy individuals and highlight the effects of blocking ET receptors in such an environmental situation.

The committee sincerely wishes full success to the authors in their future research and all other authors for the next A.L. Copley Best Paper Prize 2017.

Copley AL. The rheology of blood. A survey. J Colloid Sci 1952; 7:323.

Copley AL, Seamen GVF. The meaning of the terms rheology, biorheology and hemorheology. Clin Hemorheol 1981;1:117. doi: DOI: 10.3233/CH-1981-1202. 